UCRL-JC-118117

PREPRINT

High Power, High Beam Quality Solid State Lasers for

Materials Processing Applications

L. A. Hackel, C. B. Dane, M. R. Hermann, J. Honig,

L. E. Zapata, and M. A. Norton

This paper was prepared for submittal to the

International Congress on Applications of Lasers and Electro-Optics

Orlando, Florida, October 17-20, 1994

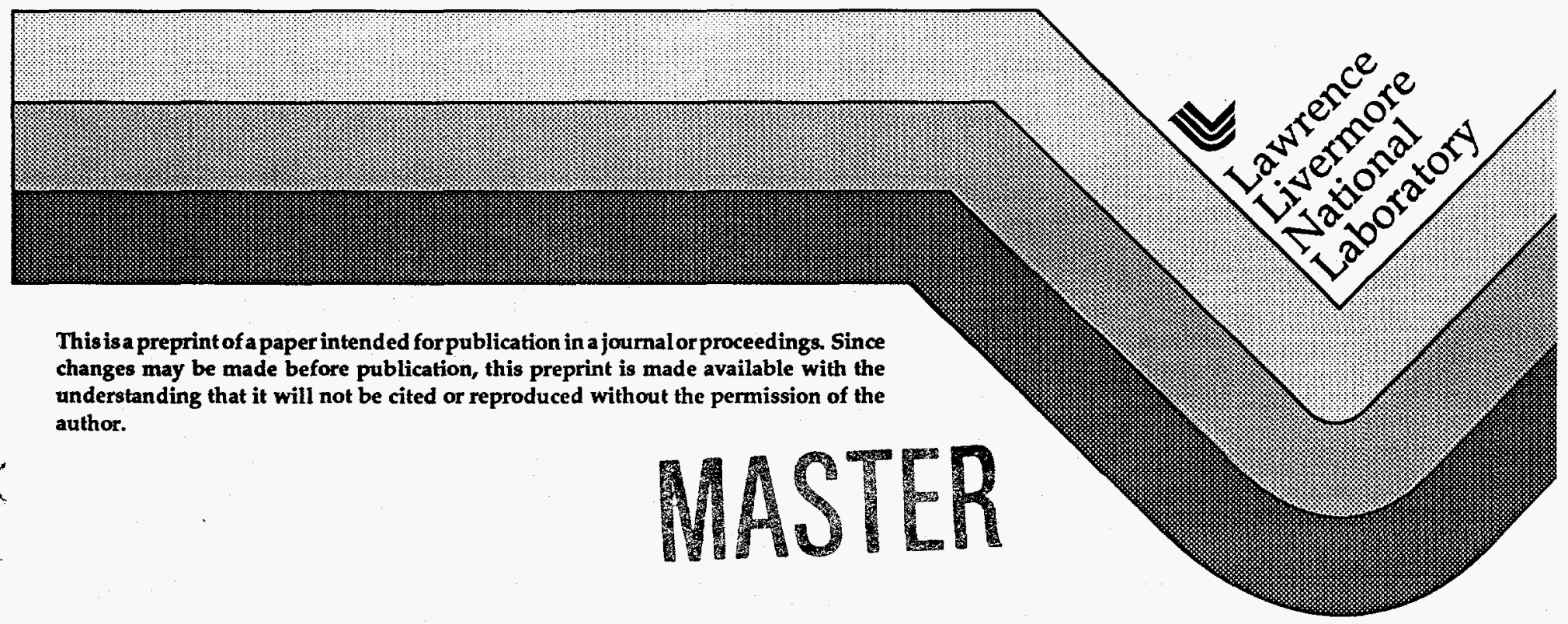




\section{DISCLAIMER}

This document was prepared as an account of work sponsored by an agency of the United States Government. Neither the United States Government nor the University of California nor any of their employees, makes any warranty, express or implied, or assumes any legal liability or responsibility for the accuracy, completeness, or usefulness of any information, apparatus, product, or process disclosed, or represents that its use would not infringe privately owned rights. Reference herein to any specific commercial products, process, or service by trade name, trademark, manufacturer, or otherwise, does not necessarily constitute or imply its endorsement, recommendation, or favoring by the United States Government or the University of California. The views and opinions of authors expressed herein do not necessarily state or reflect those of the United States Government or the University of California, and shall not be used for advertising or product endorsement purposes. 


\section{DISCLAIMER}

Portions of this document may be illegible in electronic image products. Images are produced from the best available original document. 


\title{
High Power, High Beam Quality Solid State Lasers for Materials Processing Applications
}

\author{
Lloyd A. Hackel, C. Brent Dane, Mark R. Hermann, John Honig, \\ Luis E. Zapata, and Mary A. Norton \\ Lawrence Livermore National Laboratory \\ P.O. Box 808, L-487, Livermore, CA 94550
}

\begin{abstract}
The Laser Science and Technology Department at Lawrence Livermore National Laboratory is developing solid state lasers with high average power and high beam quality. Specific systems include a laser to generate 10 to $14 \AA$ x-rays for proximity print lithography, a $400 \mathrm{~mJ}, 500 \mathrm{~Hz}$ laser for $130 \AA$ projection lithography and unique systems for speckle imaging, laser radars and medical treatments.

\section{Introduction}

The Laser Science and Technology Department of Laser Programs at Lawrence Livermore National Laboratory has a strong focus on developing solid state lasers with high average power and high beam quality. These lasers in general have characteristics which are beyond the capability of available commercial equipment and are intended to advance the state-of-the-art in a number of important applications. Specific activities in which the lasers are being employed include: proximity print $x$-ray lithography where a laser is used to generate $x$-rays in the 10 to $14 \AA$ range for printing advanced integrated circuits; extreme ultraviolet (EUV) projection lithography where a laser is used to generate $130 \AA$ radiation for even higher resolution printing of integrated circuits; cutting drilling and welding in the materials processing industry; and assorted applications that include the generation of x-rays for biological imaging, solid state lasers for $0.35 \mu \mathrm{m}$ photolithography, photo-ablation for contouring shapes and profiles in a variety of materials, speckle imaging, laser radars, and specific medical treatments.

A common thread in the architecture of most of our laser systems is a master oscillator followed by a power amplifier in which non-linear stimulated Brillouin scattering (SBS) phase conjugation is used to obtain very high beam quality. 1 Depending on the output energy and average power requirements, the laser excitation
\end{abstract}


media is Nd:glass or Nd:YAG. The lasers are pumped either with flash lamps in advanced design reflectors or by arrays of laser diodes.

The laser systems are in general developed to have unique properties not available yet in commercial devices. Examples include features such as: $30 \mathrm{~J}$ per pulse output in near-diffraction-limited beam quality and pulse lengths from 10 to $600 \mathrm{~ns}$, and $400 \mathrm{~mJ}$ per pulse output at $200 \mathrm{~Hz}$ pulse repetition rate in near-diffraction-limited beam quality. We have used specific lasers to generate over $80 \%$ doubling efficiency from 1 $\mu \mathrm{m}$ to the green at energies of $25 \mathrm{~J} /$ pulse and average powers near $100 \mathrm{~W}$. Additionally, when operated in a burst mode for a time period of 2 to 4 seconds (nominally equivalent to an IC field exposure), the laser produces up to $500 \mathrm{~W}$ of power at $1 \mu \mathrm{m}$; using the green laser output as pump, it produces up to $40 \mathrm{~W}$ average power of $x$-rays in the 10 to $14 \AA$ spectral range. We have generated third harmonic $0.355 \mu \mathrm{m}$ from the 1 $\mu \mathrm{m}$ wavelength for large area photolithography and have begun use of the diffractionlimited $400 \mathrm{~mJ}, 200 \mathrm{~Hz}$ output at $1 \mu \mathrm{m}$ for pumping a plasma to generate $130 \AA$ extreme ultraviolet light as well as its use as a high power beam for precision hole drilling in various substrates.

In this paper we discuss specifics of the laser architecture based on the master oscillator/multipass phase conjugated amplifier and how we have integrated the resulting lasers into a number of applications.

\section{Phase Conjugated Master Oscillator/Power Amplifier}

The basic design of the phase-conjugated master oscillator/power amplifier is shown in Figure 1. In this design, the output of a single-frequency master oscillator is tailored to the desired pulse length, beam quality and repetition rate and is then amplified to the desired energy in a regenerative amplifier. The exact number of passes required in the amplifier depends on the required output power and the specific gain to loss ratio of a beam transit through the amplifier. At the mid-point of the amplification, a phase conjugate mirror employing stimulated Brillouin scattering (SBS) reflects the beam back through its original path. The idea behind the conjugate mirror is that the phase error on the return path cancels the accumulated phase error on the input path and thus results in a near zero net phase error in the output beam at high power.

The master oscillator is typically operated in a self-seeded mode to produce singlefrequency, diffraction-limited output. The single frequency is required for the phase conjugator to provide low threshold and high fidelity return. The oscillator's diffraction limited output provides the reference wave front for the phase-conjugated amplification. The oscillator output is preamplified, anamorphically expanded and then imaged into the regenerative amplifier ring.

The ring amplifier is configured with a polarizer and a $90^{\circ}$ polarization rotator which act as a passive polarization switch. The input beam transits the ring once, double passing the amplifier, rotates in polarization and then passes the ring a second time. After the second ring pass, the polarization has rotated to deflect the beam to the 
phase conjugate mirror. With sufficient gain in the amplifier, the phase conjugate mirror is brought above threshold and generates a return beam which retraces the path of the input pulse. It thus results in a high power output beam with essentially all phase error removed. Due to its threshold activation, the phase conjugate mirror plays a second role as a multistage gain isolator since high input power is required to produce a reflection. The output beam having been rotated $90^{\circ}$ in polarization is separated from the input by means of the polarization rotation within the ring and the polarizer. Thus this nonlinear component inexpensively replaces Pockels cells and fast power supplies and provides the required stage to stage gain isolation.

This laser configuration is used with both glass and crystal based Nd laser systems. Per pulse output energies from the various systems range from $400 \mathrm{~mJ}$ to $30 \mathrm{~J}$ per pulse and pulse durations from 5 to $600 \mathrm{~ns}$. Glass systems have been run at repetition rates up to $6 \mathrm{~Hz}$ and the crystal systems up to $2.3 \mathrm{kHz}$. In all cases the output beam are near diffraction limited with extremely stable pointing stability. Figure 2 shows an example of the far field beam profile of the laser operated with and without the phase conjugator. The dramatic improvement provided by phase conjugation is clear.

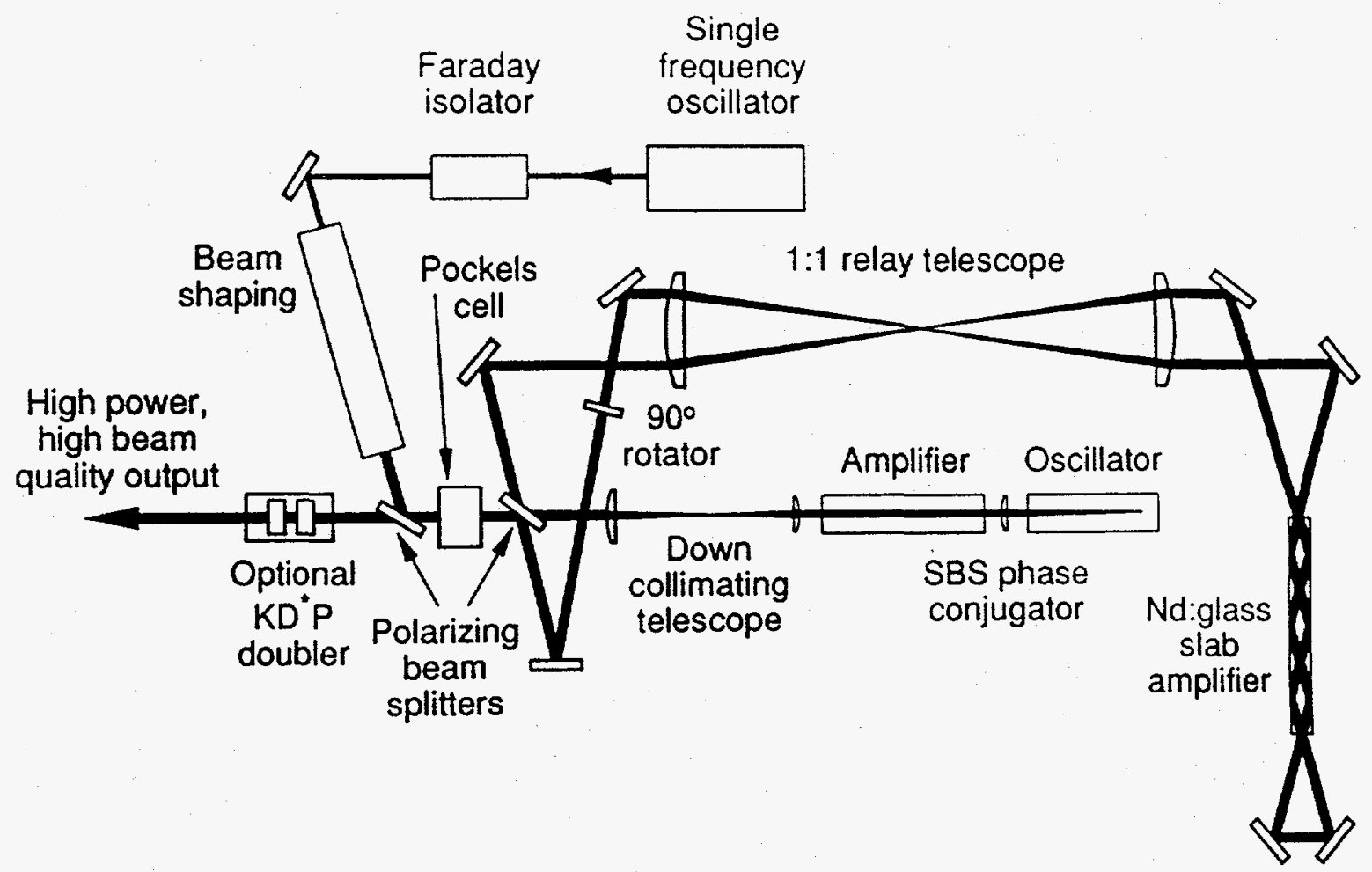

Figure 1. A schematic of the multipass amplifier geometry incorporating an SBS phase conjugate mirror. A passive $90^{\circ}$ rotator in the regenerative amplifier ring causes the injected pulse to make two ring passes (4 slab passes) before it is directed into the phase conjugator. The SBS wavefront reversed return then retraces the input path for an additional two ring passes, for a total of 8 gain passes, before it exits and is isolated from the input pathway by a Pockels cell. Other similar geometries require fewer or more passes. 
(a) Without SBS phase conjugation

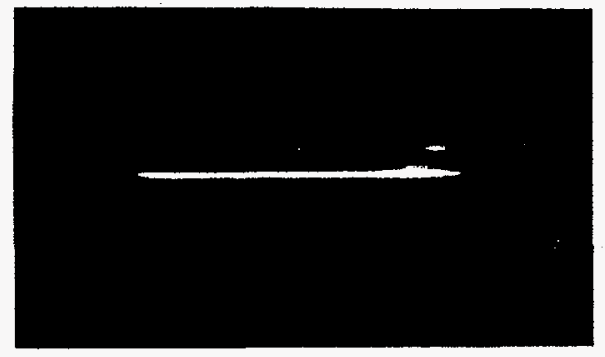

(b) With SBS phase conjugation

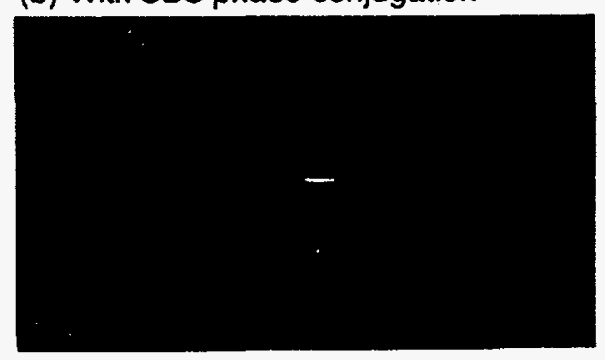

(c) Output divergence for $25 \mathrm{~J}, 3 \mathrm{~Hz}$ operation

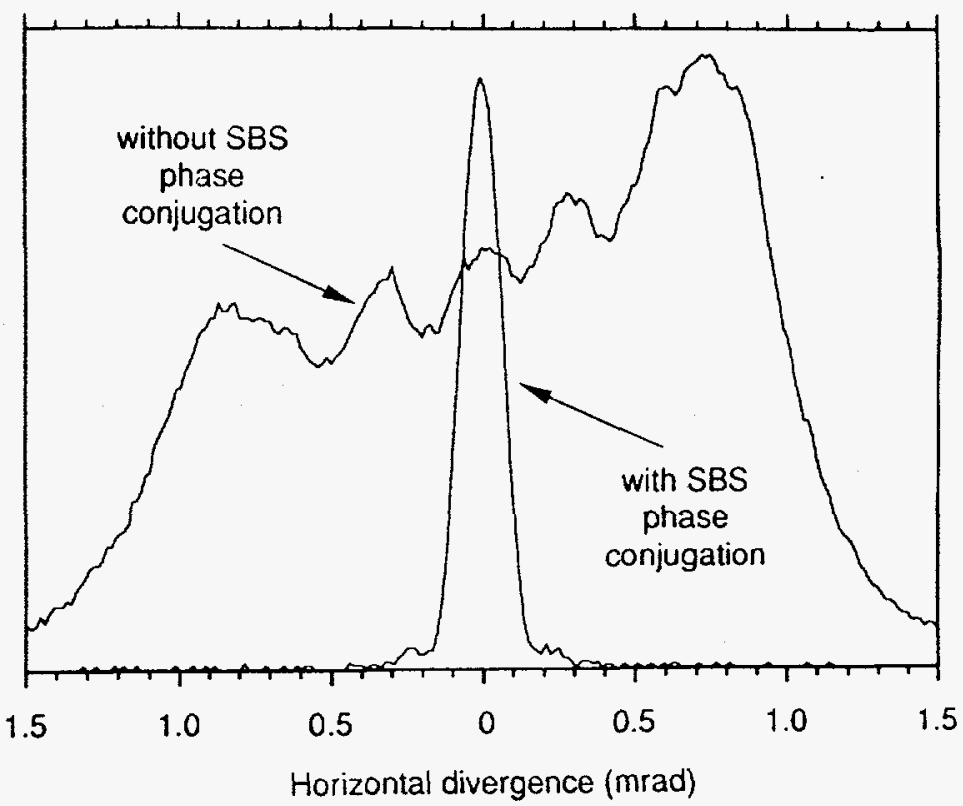

Figure 2. A comparison of the far field profiles measured at the focus of a $120 \mathrm{~cm}$ lens for $3 \mathrm{~Hz}, 75 \mathrm{~W}$ operation (a) without and (b) with SBS phase conjugation. Plot (c) overlays horizontal cross-sections of both cases.

\section{Applications of the Laser Technology}

\section{Proximity Print $X$-Ray Lithography}

Proximity print $x$-ray lithography is at the forefront of efforts to develop a commercial lithography system capable of producing circuit features of $0.25 \mu \mathrm{m}$ and smaller. A schematic drawing of the concept is shown in Figure 3 where a $25 \mathrm{~J} /$ pulse, 10 ns pulse length laser output is focused to a $\sim 50 \mu \mathrm{m}$ spot $\left(\sim 10^{14} \mathrm{~W} / \mathrm{cm}^{2}\right)$ on a metal or dense gas target. The resulting plasma generates $x$-rays in the 10 to $14 \AA$ range. Conversion efficiencies are in excess of $10 \%$. At 20 to $40 \mathrm{~cm}$ distance from this point source, the x-rays pass through a mask which is placed $\sim 40 \mu \mathrm{m}$ above a resist coated silicon wafer. The mask contains a replica of the circuit features to be printed allowing $x$-rays to pass through transparent areas and activate the exposed resist. The process is repeated on approximately 38 such fields on a typical $20 \mathrm{~cm}$ wafer followed by a development, etching and washing process to create the circuit features. For the rapid printing required in a commercial process, this technique has required advances in scanner capability to rapidly move and precisely align to each succeeding field as well 
as the development of a source capable of providing approximately $100 \mathrm{~mJ}$ of useful $\mathrm{x}$ ray output per second. This requirement translates into a laser average power of $500 \mathrm{~W}$ to $1 \mathrm{~kW}$ for a full production machine providing 40 wafer levels per hour of throughput.

Under support of the Advanced Research Projects Agency, National Lithography Program, we have developed a laboratory scale laser source operating at $150 \mathrm{~W}$ and producing $x$-rays with greater than $10 \%$ conversion efficiency. We are now in the process of building a field prototype in a two-step upgrade which will take the laser source first to $300 \mathrm{~W}$ and then to $600 \mathrm{~W}$ average power. This will be sufficient output for high throughput production demonstrations. We expect this build to be completed within the next 12 to 18 months and the equipment integrated with a stepper for high throughput testing. Following successful testing, LLNL intends to find and transfer the technology to an industrial firm for commercial manufacturing.

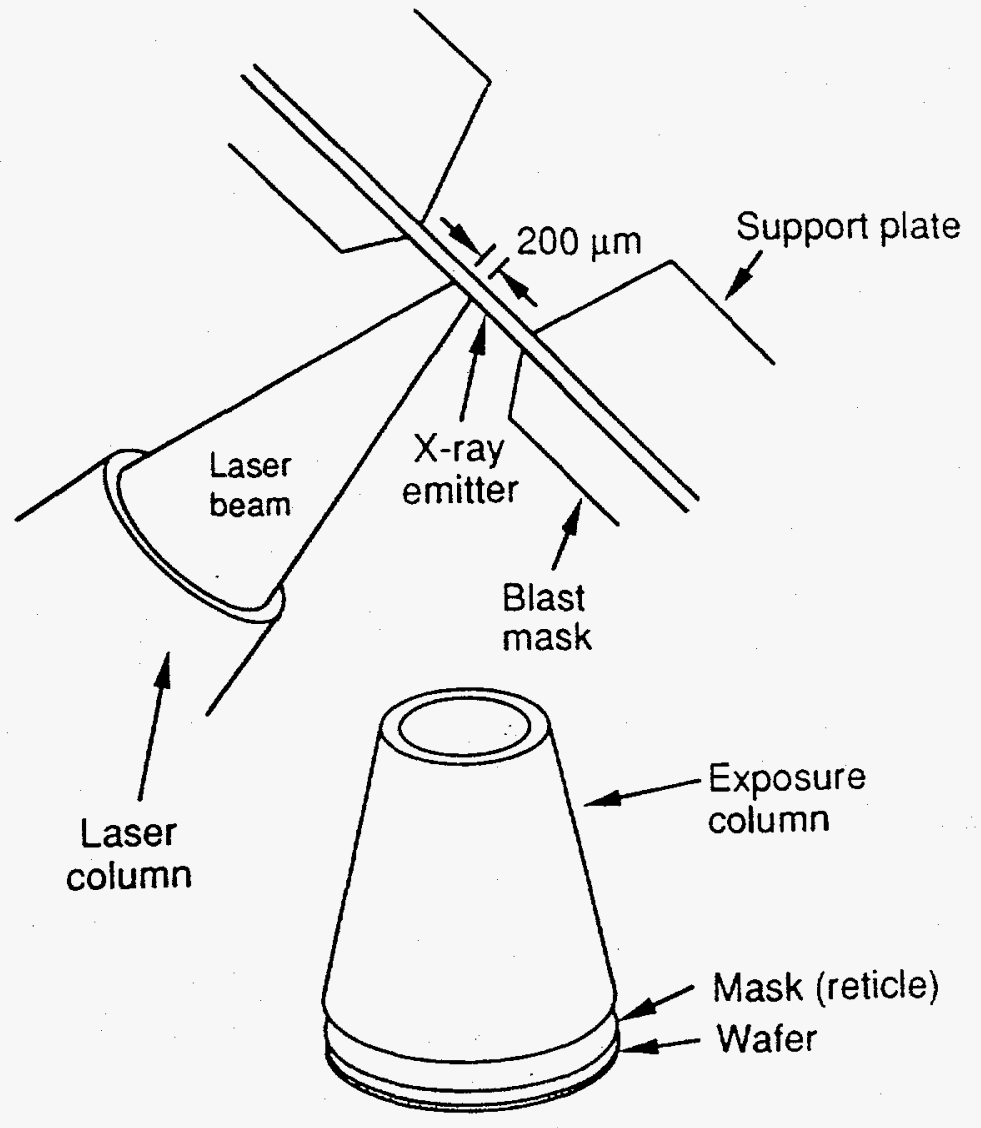

Figure 3. A schematic diagram of a proximity print x-ray lithography setup. A high energy laser is focused onto a target generating $x$-rays which pass through an exposure column and print circuit mask onto a resist coated silicon wafer. Approximately 10 to $50 \mathrm{~mJ} / \mathrm{cm}^{2}$ of $x$-ray flux is required.

\section{Extreme Ultraviolet Projection Lithography}

Projection lithography using extreme ultraviolet light offers the potential for ultrafine resolution lithography down below $0.2 \mu \mathrm{m} .2$ In the concept, schematically shown in 
Figure 4, EUV light near the $130 \AA$ region with a bandwidth of approximately $3 \AA$ is generated from a point plasma source. This wavelength choice results from the existence of high performance narrow band multilayer optics at $130 \AA$, a wavelength just longer than the silicon L-edge. The $x$-ray source is proposed as a laser produced plasma generated by the interaction of laser light of intensity greater than $1011 \mathrm{~W} / \mathrm{cm}^{2}$ with a high- $Z$ target material. As shown in the figure, EUV light from the target is efficiently collected by a pair of multilayer-coated condenser mirrors. The condenser mirrors project the narrow-band radiation onto a mask or master-reticle which works in reflection. The mask is a thin metallization pattern over a multilayer coating which, in turn, is supported by a flat, highly polished, precision, four-mirror imaging system. While there are a number of different designs for the imaging optical system, it generally can be characterized as follows: it is a ring-field imaging system providing for step and scan exposure of the wafer; an $\mathrm{f} \#$ of $f / 6$ or smaller will provide resolution of < $0.1 \mu \mathrm{m}$ at the wafer: it will project a virtually distortion-free image over the ring-field (distortion of $<0.01 \mu \mathrm{m}$ at the wafer) and it will be a demagnifying system with a reduction factor of approximately five-fold in dimension.

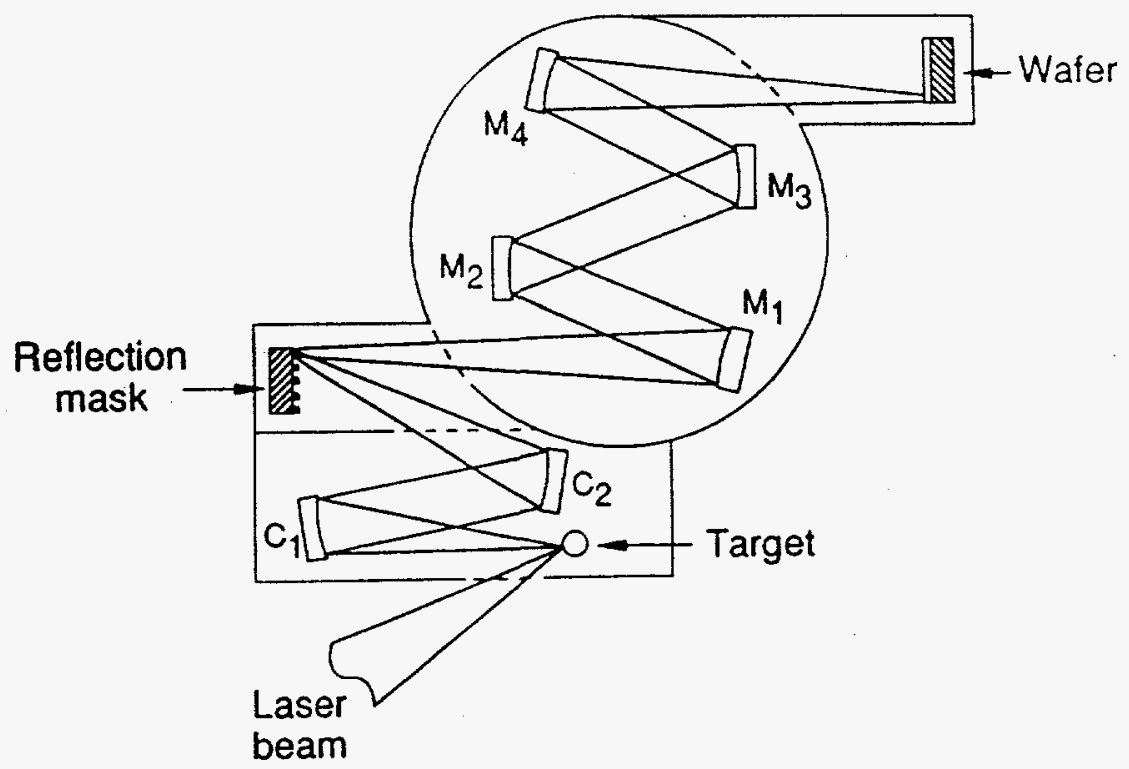

Figure 4. Schematic representation of a $5 X$ reduction, ring field EUV projection lithography system. Setup employs four imaging mirrors, a reflective mask, two condenser mirrors and is driven by a solid state laser to generate plasma produced EUV.

A significant amount of work remains to be done to field a commercial quality system. This includes the fabrication of precision aspherical mirrors and the mechanisms to align and rigidly hold them, the production of a high quality imaging resist, and an alignment and registration system capable of supporting the very fine printed features. It also involves the development of a compact, high efficiency, high average power laser source to generate the laser plasma. 
We are currently working on the development of a diode-pumped solid state laser as the source driver for the EUV lithography system. The laser for this system has been designed to a set of specifications appropriate for generating EUV radiation at $130 \AA$. The specifications of the laser are $340 \mathrm{~mJ} /$ pulse, 5 to $7.5 \mathrm{~ns}$ pulse length, and a $1.3 \mathrm{kHz}$ repetition rate.

In order to build a laser meeting these specifications, we have used a appropriately scaled version of the master oscillator/multipass amplifier technology discussed above. The oscillator for this device is a self-seeded Q-switched Nd:YAG rod pumped by an array of laser diodes. The power amplifier is also Nd:YAG in the form of a slab pumped by two large arrays of laser diodes. The slab has a gain of 4 per pass (1.4 nepers). As in the previously described design, high beam quality and high extraction efficiency will be maintained in this device through the use of an SBS phase conjugator placed at the mid-transit point in the amplifier. The amplifier is configured to use passive polarization and 8 amplifier passes for efficient extraction.

The high repetition rate of the laser diode pumps is achieved through the use of an aggressive heat removal method using silicon microchannel coolers. 3 These coolers can maintain acceptable diode operating temperatures while rejecting heat at rates up to 1 $\mathrm{kW} / \mathrm{cm}^{2}$ Each cooler consists of a silicon package on which two $0.9 \mathrm{~mm}$ long diode bars are mounted. These are then stacked at a density of 10 packages per $\mathrm{cm}$ with a single water inlet and outlet. Each package requires a cooling flow of $1 \mathrm{~cm} 3 / \mathrm{s}$ with a pressure of $60 \mathrm{psi}$. In this configuration each $1.8 \mathrm{~cm}$ wide diode package is capable of $100 \mathrm{~W}$ peak power output. No reduction in this peak power is observed at a duty cycle of up to $25 \%$ resulting in an average power of $25 \mathrm{~W} /$ package and a diode array average pumping irradiance of $140 \mathrm{~W} / \mathrm{cm}^{2}$.

\section{Coherent Laser Radar}

The high power laser systems developed by means of the phase-conjugated master oscillator/multipass power amplifier display two properties often critically important to laser radar applications; they have long coherence lengths with the absence of frequency chirp and the output beam quality is nearly diffraction-limited. The long coherence length results from the single frequency nature of the master oscillator and the preservation of this narrow bandwidth in the stimulated scattering process of phase conjugation. Coherence lengths near the transform limit are generally expected. Figure 5 shows an example in which full contrast fringes are generated in a $15 \mathrm{~J}$ per pulse output of a $600 \mathrm{~ns}$ long pulse. The second important attribute for radar applications is the neardiffraction-limited beam quality. As discussed above the low power master oscillator can rather easily be operated in a diffraction-limited TEM 00 mode and the phase conjugation process restores this high quality wavefront to the amplified beam. High beam quality ensures maximum energy on target and hence maximum radar return signals. 


\section{Laser Machining}

The high power and high beam quality make this laser system ideally suited for laser machining applications. Various configurations of this laser architecture result in a range of laser parameters important for different applications. One configuration utilizing Nd:glass slabs can produce per pulse energies up to $30 \mathrm{~J}$ with pulse lengths adjustable from $10 \mathrm{~ns}$ to $1 \mu \mathrm{s}$ and repetition rates up to $10 \mathrm{~Hz}$, all with near-diffractionlimited focus control. In another configuration employing Nd:YAG crystal slabs the 400 $\mathrm{mJ}$ per pulse can be generated at rates up to and exceeding $3 \mathrm{kHz}$, again with diffraction-limited beam quality.
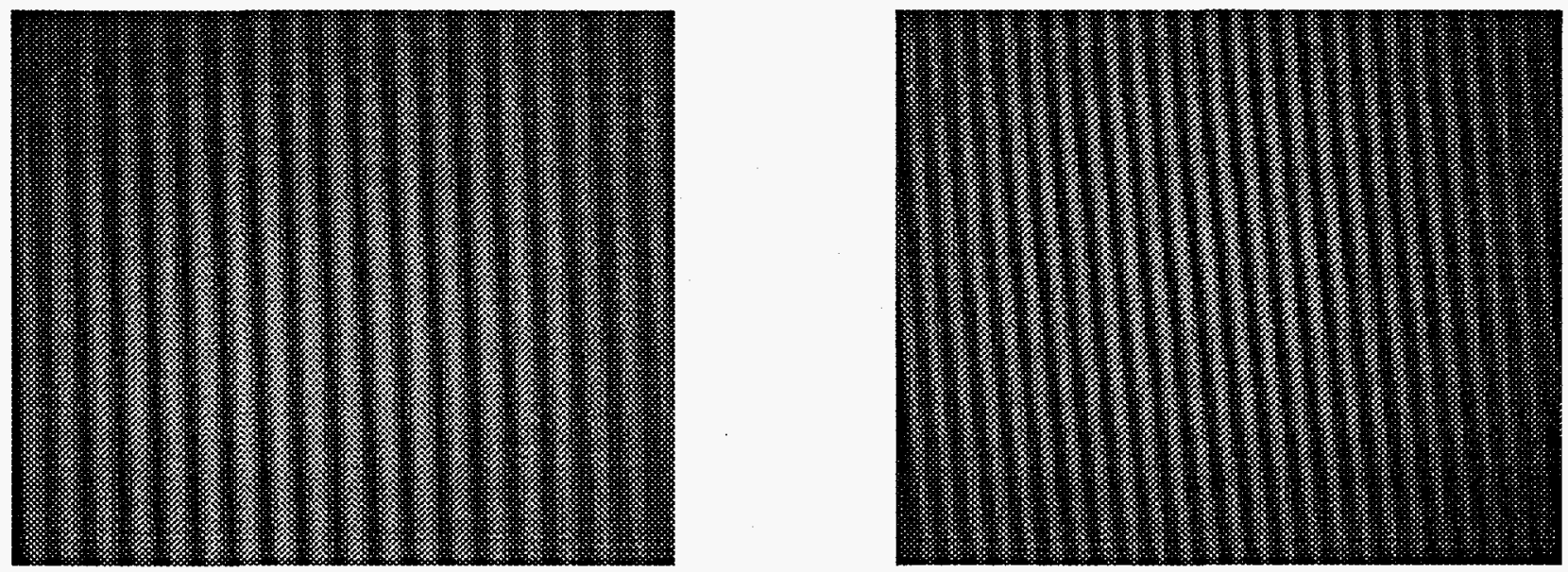

Figure 5. High fringe visibility is observed for (left) equal fiber pathlengths and (right) a fiber pathlength of $20 \mathrm{~m}$. A fiber index of $\sim 1.5$ translates into a pathlength difference of $30 \mathrm{~m}$ equivalent. The sampled light was the 15 $\mathrm{J} /$ pulse, $600 \mathrm{~ns}$ pulse length output of the $\mathrm{Nd}$ :glass laser system.

\section{Summary}

The technology of advanced solid state lasers employing a master oscillator/phase conjugated power amplifier architecture is being developed which can be useful in a broad range of industrial applications. We believe that the high level of performance from these lasers can be maintained in field situations.

\section{Acknowledgments}

This work was performed under the auspices of the U.S. Department of Energy by Lawrence Livermore National Laboratory under contract W-7405-Eng-48. Aspects of the work were supported by the ARPA National Lithography Program. 


\section{References}

1. L. A. Hackel, J. L. Miller, and C. B. Dane, "Phase Conjugation in a High Power Regenerative Laser Amplifier System," Int. J. Nonlin. Opt. Phys. 2, 171-185 (1993).

2. A. M. Hawryluk and L. G. Seppala, "Soft X-Ray Projection Lithography Using an $X-$ Ray Reduction Camera," J. Vac. Sci. Tech. B6, 2162-2166 (1988). Also, W. T. Silfvast and O. R. Wood II, "Tenth Micron Lithography with a $10 \mathrm{~Hz} 37.2 \mathrm{~nm}$ Sodium Laser," Microelectron. Eng. 8, 3-11 (1988).

3. D. Mundinger, R. Beach, W. Benett, R. Solarz, W. Krupke, R. Staver, and D. Tuckerman, "Demonstration of High Performance Silicon Microchannel Heat Exchangers for Laser Diode Array Cooling," Appl. Phys Lett. 53, 1030-1032 (1988). 